\title{
Evaluación de la mineralización de biosólidos de plantas de tratamiento de aguas residuales domésticas
}

\author{
Jorge A. Silva ${ }^{1}$, Patricia Torres L. ${ }^{1} \&$ Jaime Mosquera R. ${ }^{1}$
}

\begin{abstract}
RESUMEN
Los biosólidos procedentes del tratamiento de aguas residuales municipales tienen alto potencial de aplicación agrícola por sus contenidos de materia orgánica y nutrientes. En esta investigación se evaluó la mineralización de los biosólidos provenientes de la Planta de Tratamiento de Aguas Residuales de Cañaveralejo, Cali- Colombia, mediante el ajuste de modelos de regresión; se realizó el seguimiento del contenido de $\mathrm{NH}_{4}{ }^{+}, \mathrm{NO}_{3}^{-}$y $\mathrm{NO}_{2}$ - durante 126 días para tres tipos de biosólidos: deshidratado, secado térmicamente y alcalinizado, los cuales fueron aplicados a un suelo Vertic Endoaquepts en dosis de 35.4, 36.4 y $54.5 \mathrm{t} \mathrm{ha}^{-1}$ respectivamente. Para el ajuste de las curvas de regresión, se consideraron los modelos simple exponencial, doble exponencial, hiperbólico, parabólico y algunos modelos estadísticos regulares. Los resultados mostraron que el modelo parabólico propuesto por Broadbent presentó el mejor ajuste para describir el proceso de mineralización del suelo evaluado; el modelo exponencial de Stanford \& Smith, se mostró como una segunda opción de modelación, permitiendo corroborar el incremento del nitrógeno orgánico $\mathrm{N}_{0}$ cuando se realiza la aplicación de los biosólidos y el incremento de la mineralización con relación al tratamiento testigo.
\end{abstract}

Palavras-chave: nitrógeno mineralizado, fracción de mineralización, modelo parabólico

\section{Evaluation of mineralization rates of biosolids from domestic wastewater treatment plants}

\begin{abstract}
The biosolids from wastewater treatment plants have high potential of agricultural application due to the contents of organic matter and nutrients. The mineralization of biosolids from the Cañaveralejo Wastewater Treatment Plant, Cali-Colombia, was evaluated by fitting regression models. The content of $\mathrm{NH}_{4}{ }^{+}, \mathrm{NO}_{3}{ }^{-}$ and $\mathrm{NO}_{2}{ }^{-}$were monitored during 126 days for three types of biosolids: dehydrated, thermally dried and alkalinized, which were applied to a Vertic Endoaquepts soil using doses of 35.4, 36.4 and $54.5 \mathrm{t} \mathrm{ha}^{-1}$ respectively. To adjust the regression curves, the models used were: simple exponential, double exponential, hyperbolic, parabolic and some regular statistical models. The results showed that the parabolic model proposed by Broadbent presented the best fit to describe the process of mineralization in the evaluated soil. The exponential model of Stanford \& Smith was the second option of modeling, permitting corroborate the increase of organic nitrogen $\left(\mathrm{N}_{0}\right)$ when the biosolids are applied and the mineralization increase in relation to the control treatment.
\end{abstract}

Key words: mineralized nitrogen, mineralization rates, parabolic model 


\section{INTRODUCCIÓN}

El nitrógeno es el nutriente de mayor requerimiento por parte de los diferentes cultivos, su alto costo y las grandes cantidades requeridas lo han ubicado como el limitante en la producción agrícola mundial. En el suelo, el nitrógeno se encuentra predominantemente en formas orgánicas como un componente de los diferentes compuestos y comparado con otros nutrientes, sufre diferentes transformaciones en el suelo, lo cual hace compleja la evaluación de su disponibilidad para el consumo de las plantas (Pereira et al., 2005).

La mineralización de nitrógeno es el proceso biológico mediante el cual el nitrógeno orgánico es transformado a formas inorgánicas o mineralizadas como amonio $\left(\mathrm{NH}_{4}^{+}\right)$, nitrato $\left(\mathrm{NO}_{3}^{-}\right)$ y nitrito $\left(\mathrm{NO}_{2}^{-}\right)$, las cuales forman el nitrógeno disponible para las plantas y los microorganismos del suelo (Bettiol \& Camargo, 2008). La cantidad de nitrógeno aplicada sobre el suelo no debe exceder los requerimientos del cultivo puesto que se corre el riesgo de superar la capacidad de absorción de las raíces de las plantas, lo que puede facilitar que este elemento lixivie y contamine cuerpos de agua subterráneas (Bettiol \& Camargo, 2008; Dynia et al., 2006).

Uno de los criterios usados para calcular la dosis de biosólido a aplicar en el cultivo, sugiere evaluar la cantidad de nitrógeno disponible en este material, la cual se define como la suma del nitrógeno mineral $\left(\mathrm{NH}_{4}^{+}, \mathrm{NO}_{3}^{-}\right.$y $\left.\mathrm{NO}_{2}^{-}\right)$contenido en el biosólido y la fracción del nitrógeno orgánico que puede ser mineralizada durante el ciclo del cultivo, conocida como la fracción potencial de mineralización (Boeira, 2004), la cual puede ser estimada mediante la incubación de una masa de suelo conocida, bajo condiciones de temperatura y humedad controladas por tiempos definidos, determinando el incremento de nitrógeno inorgánico al finalizar el periodo de incubación (Ortega \& Mardonez, 2005).

El comportamiento de la fracción de mineralización depende directamente del tipo de tratamiento o proceso de estabilización de los biosólidos, duración del proceso y tipo de materia orgánica del biosólido (Bettiol \& Camargo, 2008). Esta fracción de mineralización puede disminuir a medida que se aumenta la dosis, generando un riesgo ambiental negativo por la lixiviación de nitratos (Boeira, 2009).

A través del uso de modelos empíricos es posible predecir los fenómenos de mineralización que ocurren en la naturaleza mediante el ajuste de ecuaciones matemáticas a los datos experimentales obtenidos en los procesos de incubación (Camargo et al., 2002). Los primeros en describir dichas ecuaciones fueron Stanford \& Smith (1972) quienes postulan que la dinámica de mineralización del nitrógeno orgánico en suelos nativos puede ser descrita por una cinética de primer orden Ec. 1.

$$
\mathrm{N}_{\mathrm{t}}=\mathrm{N}_{\mathrm{o}}\left(1-\mathrm{e}^{-\mathrm{kt}}\right)
$$

$\mathrm{N}_{\mathrm{t}} \quad$ - nitrógeno mineralizado en el tiempo $\mathrm{t}$

No - nitrógeno orgánico potencialmente mineralizable $(\mathrm{t} \rightarrow \infty)$

$\mathrm{k} \quad$ - constante de mineralización $\left(\mathrm{d}^{-1}\right)$

t - tiempo
$\mathrm{Al}$ emplear este modelo matemático, se asume que bajo ciertas condiciones ambientales la fracción de mineralización del nitrógeno es proporcional a la cantidad de sustrato mineralizable en el suelo; los parámetros obtenidos con este modelo son útiles en la definición del balance entre las necesidades del cultivo y el nitrógeno aportado por el biosólido aplicado al suelo (Boeira \& Maximiliano, 2008). Autores como Jones (1984); Cabrera (1993) y Cabrera et al. (2005) incluyeron en este modelo simple la influencia de la humedad en el proceso de mineralización, adicionando una segunda constante para describir este efecto Ec. 2 o un componente lineal Ec. 3 respectivamente.

$$
\begin{aligned}
& \mathrm{N}_{\mathrm{t}}=\mathrm{N}_{1}+\mathrm{N}_{\mathrm{o}}\left(1-\mathrm{e}^{-\mathrm{kt}}\right) \\
& \mathrm{N}_{\mathrm{t}}=\mathrm{N}_{\mathrm{o}}\left(1-\mathrm{e}^{-\mathrm{k}_{2} \mathrm{t}}\right)+\mathrm{k}_{1} \mathrm{t}
\end{aligned}
$$

$\mathrm{N}_{1} \quad$ - nitrógeno mineralizado presente en el suelo al inicio de la observación, $\mathrm{t}=0$

No - nitrógeno orgánico potencialmente mineralizable, $\mathrm{t} \rightarrow \infty$

$\mathrm{k}, \mathrm{k}_{2}$ - constante de mineralización exponencial, $\mathrm{d}^{-1}$

$\mathrm{k}_{1} \quad$ - constante de mineralización, $\mathrm{d}^{-1}$

t - tiempo

Juma et al. (1984) encontraron que los datos de nitrógeno mineralizado presentaban una tendencia curvilínea, sobre la cual plantearon un modelo hiperbólico para describir dichas transformaciones, realizando una estimación de la vida media de la mineralización Ec. 4.

$$
\mathrm{N}_{\mathrm{t}}=\mathrm{N}_{\mathrm{o}}\left(\frac{\mathrm{t}}{\sqrt{\mathrm{t}}+\mathrm{t}}\right)=\mathrm{N}_{\mathrm{o}}\left(\frac{\sqrt{\mathrm{t}}}{1+\sqrt{\mathrm{t}}}\right)
$$

No - nitrógeno orgánico potencialmente mineralizable, $\mathrm{t} \rightarrow \infty$

t - tiempo

Broadbent (1986) encontró que el modelo simple exponencial no se ajusta a la realidad, por lo que propuso un modelo del orden parabólico, el cual proporcionó un mejor ajuste a sus datos experimentales Ec. 5 .

$$
\mathrm{N}_{\mathrm{t}}=A \mathrm{t}^{\mathrm{b}}
$$

\section{A y b - constantes}

Molina et al. (1980) e Inubushi et al. (1985) encontraron que el modelo de Stanford \& Smith (1972) no describe de manera satisfactoria la cinética de mineralización neta y consideraron la existencia de dos fracciones orgánicas en el proceso de mineralización de nitrógeno, una que puede ser rápidamente transformada y otra más recalcitrante Ec. 6.

$$
\mathrm{N}_{\mathrm{t}}=\mathrm{N}_{\mathrm{oq}}\left(1-\mathrm{e}^{-\mathrm{k}_{\mathrm{q}} \mathrm{t}}\right)+\mathrm{N}_{\mathrm{os}}\left(1-\mathrm{e}^{-\mathrm{k}_{\mathrm{s}} \mathrm{t}}\right)
$$

$\mathrm{N}_{\mathrm{t}} \quad$ - nitrógeno mineralizado en el tiempo $\mathrm{t}$ 
Noq - nitrógeno orgánico potencialmente mineralizado zona rápida

Nos - nitrógeno orgánico potencialmente mineralizado zona recalcitrante

$\mathrm{k}_{\mathrm{a}} \mathrm{y} \mathrm{k}_{\mathrm{S}}$ - constantes de las tasas de mineralización para cada zona

t $\quad$ - tiempo

Estos modelos doble exponencial fueron usados por Smith et al. (1998) y Wang et al. (2004) mostrando buenos resultados para la descripción de la mineralización en un suelo y biosólidos incubados bajo condiciones constantes de temperatura y humedad. Sin embargo, Dou et al. (1996) encontraron que el ajuste de los modelos dependía de la duración de incubación, ya que a las 30 semanas de incubación el ajuste del modelo doble exponencial fue significativamente mejor al de un modelo exponencial simple e hiperbólico; para ensayos con tiempos de incubación menores a 30 semanas, recomiendan el ajuste de un modelo del orden simple exponencial para representar la dinámica del proceso de mineralización. Adicionalmente, Flores et al. (2007) encontraron que los valores de mineralización bajos (4 y 7\%) del biosólido alcalinizado pudieron estar afectados por la temperatura del suelo la cual fue menor a $15^{\circ} \mathrm{C}$.

Teniendo en cuenta que no se presenta un acuerdo entre la existencia de un modelo óptimo capaz de describir el proceso de mineralización en todo el tiempo de incubación y conociendo que dicho proceso está influenciado por diferentes características como tipo de suelo y de biosólido, temperatura y humedad, esta investigación evaluó diferentes modelos de regresión para determinar el que mejor describa la mineralización de los biosólidos generados en la Planta de Tratamiento de Aguas Residuales de Cañaveralejo PTAR-C de la Ciudad de Cali, Colombia.

\section{Material y Metodos}

El experimento fue desarrollado sobre un suelo vertic endoaquepts empleado regularmente para el cultivo de caña de azúcar. Los biosólidos evaluados fueron tomados de la PTAR-C de Cali-Colombia, los cuales son digeridos por vía anaerobia y deshidratados en filtros prensa (BD). Debido a la presencia de patógenos en este biosólido, se realizaron procesos complementarios de reducción de patógenos mediante secado térmico $\left(60{ }^{\circ} \mathrm{C}\right.$ por $\left.12.58 \mathrm{~h}\right)(\mathrm{BDST}) \mathrm{y}$ tratamiento alcalino (cal viva $9 \%$ peso seco) (BDTA) de acuerdo con lo reportado por Silva et al. (2012). El experimento incluyó un tratamiento control que mostró el proceso de mineralización normal del suelo. La Tabla 1 presenta los tratamientos evaluados y las dosis aplicadas en cada uno de los casos.

El estudio fue ejecutado en escala de laboratorio bajo un arreglo completamente al azar con 2 repeticiones por tratamiento y 2 dosis: D0 suelo (control), D1: dosis requerida por el cultivo (caña de azúcar). Los biosólidos fueron mezclados con $280 \mathrm{~g}$ de suelo y la humedad se mantuvo al $70 \%$ de la capacidad de campo del suelo según CETESB (1999).

En todos los tratamientos y sus respectivos duplicados fue cuantificado el contenido de nitrógeno mineralizado $\left(\mathrm{NH}_{4}^{+}\right.$,

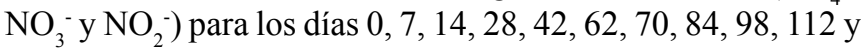

Tabla 1. Tratamientos y dosis empleadas

\begin{tabular}{|c|c|c|}
\hline Tratamiento & $\begin{array}{c}\text { Tasa } \\
\text { aplicada } \\
\left(\mathrm{t} \mathrm{ha}^{-1}\right)^{*}\end{array}$ & $\begin{array}{c}\text { Tasa aplicada } \\
\left(\mathrm{mg} \mathrm{N} \text { orgánico } \mathrm{kg}^{-1}\right)^{* *}\end{array}$ \\
\hline Control & 0 & 0 \\
\hline Biosólido deshidratado (BD) & 35.8 & 319.4 \\
\hline Biosólido secado térmicamente (BDST) & 36.4 & 343.8 \\
\hline Biosólido alcalinizado (BDTA) & 54.5 & 374.0 \\
\hline
\end{tabular}

126 desde la aplicación del biosólido. Con los valores promedio de nitrógeno mineral se realizaron representaciones gráficas de la evolución del contenido del nitrógeno del suelo en el tiempo con gráficos de dispersión (tiempo incubación vs mineralización media), sobre los cuales se determinó una primera aproximación para los parámetros de los modelos evaluados contrastando los valores observados y el modelo ajustado de forma simultánea a través de herramientas de hoja de cálculo Excel 2007 ® hasta obtener un ajuste visual aceptable.

Los parámetros ajustados de forma visual fueron usados como estimaciones preliminares, las cuales alimentaron el proceso de estimación final para cada uno de los modelos evaluados. Adicional a los modelos anteriormente descritos, se propuso el ajuste de tres modelos estadísticos habituales: lineal, polinomial de orden 2 y exponencial, conformando la galería de modelos que se presentan en la Tabla 2.

Tabla 2. Modelos propuestos para el ajuste de los datos

\begin{tabular}{|c|c|c|}
\hline Modelo & Ecuación & Autor \\
\hline \multicolumn{3}{|c|}{ Modelos no lineales } \\
\hline \multirow{3}{*}{\multicolumn{2}{|c|}{$\begin{array}{l}\text { a. Simple exponencial } N_{t}=N_{o}\left(1-e^{-k t}\right) \\
\text { b. Simple exponencial } N_{t}=N_{1}+N_{o}\left(1-e^{-k t}\right) \\
\text { c. Simple exponencial } N_{t}=N_{o}\left(1-e^{-k_{2} t}\right)+k_{1} t \\
N_{t}=\frac{N_{o} t}{}\end{array}$}} & \multirow{3}{*}{$\begin{array}{c}\text { Stanford \& Smith (1972) } \\
\text { Jones (1984) } \\
\text { Cabrera (1993) }\end{array}$} \\
\hline & & \\
\hline & & \\
\hline d. Hiperbólico & $\mathrm{N}_{\mathrm{t}}=\frac{\mathrm{N}_{\mathrm{o}} \mathrm{t}}{(\sqrt{\mathrm{t}}+\mathrm{t})}$ & Juma et al. (1984) \\
\hline \multicolumn{3}{|c|}{ Modelos lineales /inealizables } \\
\hline Parabólico & $\mathrm{N}_{\mathrm{t}}=A \mathrm{t}^{\mathrm{b}}$ & Broadbent (1986) \\
\hline Lineal & $\mathrm{N}_{\mathrm{t}}=\beta_{\mathrm{o}}+\beta_{1} \mathrm{t}$ & - \\
\hline g. Polinomial (2) & $N_{t}=\beta_{o}+\beta_{1} t+\beta_{2} t^{2}$ & - \\
\hline h. Exponencial & $\mathrm{N}_{\mathrm{t}}=A \mathrm{e}^{\mathrm{kt}}$ & - \\
\hline
\end{tabular}

La estimación final de los parámetros para los modelos no lineales ( $\mathrm{a}, \mathrm{b}, \mathrm{c}$ y d) se llevó a cabo mediante la implementación del algoritmo de Newton Raphson disponible en la función nls, de la librería Stats del software estadístico R.2.10, útil en la estimación de parámetros de regresión en este tipo de modelos. El ajuste de los modelos linealizables (e, f, g, h) se realizó mediante procedimientos de estimación por mínimos cuadrados, implementados en la función lm de la misma librería Stats en el Software R.2.10.

Para cada modelo ajustado se obtuvieron sus correspondientes medidas de bondad de ajuste, Coeficiente de Determinación $\left(\mathrm{R}^{2}\right)$ y el Cuadrado Medio del Error (CME), identificando el modelo de mejor ajuste como aquel que maximiza el coeficiente $\mathrm{R}^{2} \mathrm{o}$ minimiza el CME.

\section{RESUltadOS y Discusión}

La Figura 1 representa gráficamente la evolución observada en el proceso de mineralización del nitrógeno en el suelo durante los 
126 días del ensayo para cada uno de los tratamientos aplicados sobre el biosólido. El contenido de nitrógeno mineralizado del suelo fue descontado para efecto de la representación gráfica, razón por la cual el día 0 se representa con un valor de 0.

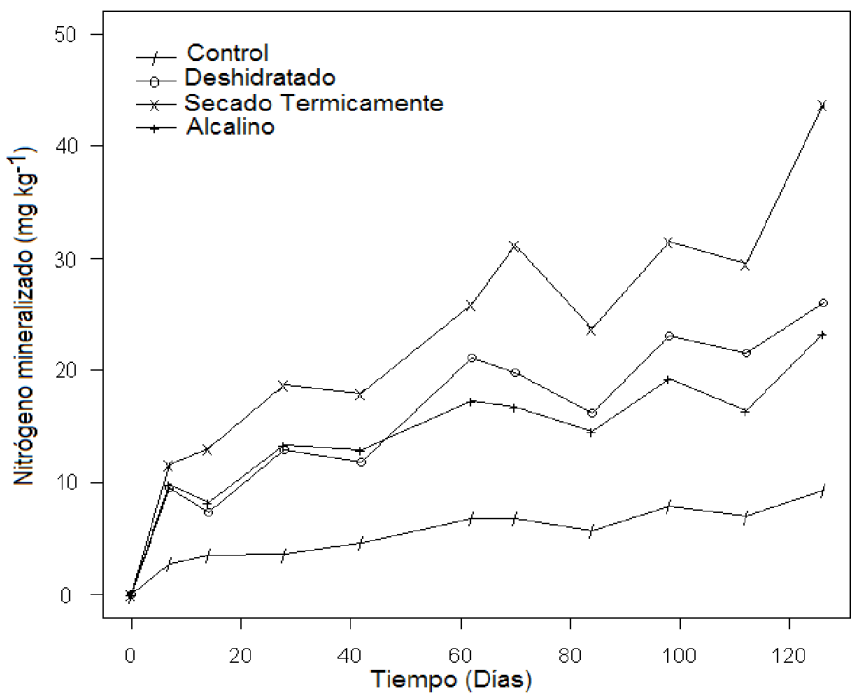

Figura 1. Disponibilidad de nitrógeno mineralizado $\left(\mathrm{NH}_{4}{ }^{+}, \mathrm{NO}_{3}{ }^{-}\right.$y $\left.\mathrm{NO}_{2}{ }^{-}\right)$durante los 126 días del ensayo para los tratamientos evaluados

Los resultados muestran un incremento en el contenido de nitrógeno mineralizado en cada uno de los tratamientos evaluados con relación al tratamiento control, presentándose un comportamiento similar para el biosólido deshidratado y el alcalinizado. El biosólido secado térmicamente mostró un mayor incremento del nitrógeno mineralizado a lo largo del periodo de incubación, debido probablemente a que el secado térmico reduce la población microbiana, la cual se restaura en el momento de la aplicación al suelo, acelerando el proceso de mineralización (Kieft et al., 1987). Los aumentos del nitrógeno mineralizado para todos los tratamientos variaron entre 6.37 a $34.3 \mathrm{mg}$ del nitrógeno mineralizado por $\mathrm{kg}$ con relación al tratamiento control para cada tiempo.

En el caso del biosólido deshidratado aplicado en una dosis de $35.8 \mathrm{t} \mathrm{ha}^{-1}$, acumulando el valor del nitrógeno mineralizado para los 126 días de seguimiento, éste corresponde a $111 \mathrm{mg}$ $\mathrm{kg}^{-1}$, valor similar a lo encontrado por Hernández et al. (2002) quienes evaluaron un biosólido deshidratado con dosis de 60 y $100 \mathrm{t} \mathrm{ha}^{-1}$ encontrando aumentos de nitrógeno mineralizado acumulado entre $200-300 \mathrm{mg} \mathrm{kg}^{-1}$.

El biosólido secado térmicamente mostró un valor de nitrógeno mineralizado acumulado de $188 \mathrm{mg} \mathrm{kg}^{-1}$, el cual fue menor a los valores reportados por Smith \& Durham (2002) quienes encontraron mineralizaciones de nitrógeno de 63-120 mg $\mathrm{N} \mathrm{kg}^{-1}$ empleando dosis en promedio de 0.43 t ha ${ }^{-1}$ para un biosólido secado térmicamente; estos valores fueron mayores debido probablemente a que la incubación se realizó con temperatura controlada y ausencia total de luz. Sin embargo, los valores de nitrógeno acumulados del biosólido secado térmicamente fueron mayores comparados con los otros biosólidos evaluados, lo que confirma lo encontrado por Silva et al. (2012) quienes encontraron que el proceso de secado térmico influye en el contenido de nitrógeno mineralizado en el suelo. Con relación al biosólido alcalino, éste mostró valores de nitrógeno mineral acumulado en los 126 días de $93.7 \mathrm{mg}$ $\mathrm{kg}^{-1}$, valor que es similar a lo reportado por Franco et al. (2003) quienes emplearon $56 \mathrm{t} \mathrm{ha}^{-1} \mathrm{y}$ encontraron valores cercanos a $100 \mathrm{mg} \mathrm{kg}^{-1}$ de nitrógeno durante 70 días de seguimiento.

La Tabla 3 muestra los resultados de la modelación sobre el tratamiento control (suelo sin aplicación de biosólido).

Tabla 3. Resultados de la modelación sobre el tratamiento control

\begin{tabular}{|c|c|c|c|c|}
\hline Modelo & Parámetros & Estimación & $\mathbf{R}^{2}$ & CME \\
\hline a. Stanford \& Smith (1972) & $\begin{array}{c}\mathrm{N}_{0} \\
\mathrm{k}\end{array}$ & $\begin{array}{l}8,2900 \\
0,0240\end{array}$ & 0,8692 & 1,04 \\
\hline b. Jones (1984) & $\begin{array}{l}\mathrm{N}_{1} \\
\mathrm{~N}_{0} \\
\mathrm{k}\end{array}$ & $\begin{array}{r}* 1,0900 \\
8,4400 \\
* 0,0140\end{array}$ & 0,8939 & 0,95 \\
\hline c. Cabrera (1993) & $\begin{array}{l}\mathrm{N}_{0} \\
\mathrm{k}_{1} \\
\mathrm{~K}_{2}\end{array}$ & $\begin{array}{r}2,8400 \\
* 0,2940 \\
0,0470 \\
\end{array}$ & 0,9318 & 0,61 \\
\hline d. Juma et al. (1984) & $\mathrm{N}_{0}$ & 6,8400 & 0,3015 & 2,85 \\
\hline e. Broadbent (1986) & $\begin{array}{l}A \\
b\end{array}$ & $\begin{array}{l}0,9770 \\
0,4430\end{array}$ & 0,9250 & 0,59 \\
\hline f. Lineal & $\begin{array}{l}\beta_{0} \\
\beta_{1}\end{array}$ & $\begin{array}{l}1,9690 \\
0,0560 \\
\end{array}$ & 0,8564 & 1,14 \\
\hline g. Polinomial (2) & $\begin{array}{l}\beta_{0} \\
\beta_{1} \\
\beta_{2}\end{array}$ & $\begin{array}{r}1,3740 \\
0,0930 \\
-0,0003 \\
\end{array}$ & 0,8852 & 1,03 \\
\hline h. Exponencial & $\begin{array}{l}A \\
k\end{array}$ & $\begin{array}{l}2,7980 \\
0,0096\end{array}$ & 0,7858 & 1,70 \\
\hline
\end{tabular}

De acuerdo con la Tabla 3, los modelos propuestos por Jones (1984) y Cabrera (1993) presentan un ajuste importante a la dinámica de mineralización natural (sin intervención) de este tipo de suelo $\left(\mathrm{R}^{2}>0,89\right)$; sin embargo, al evaluar el aporte particular de cada uno de sus parámetros, se encuentra falta de significancia del parámetro $\mathrm{N}_{1}$ para el caso del modelo de Jones (1984) y k en $_{1}$ el caso del modelo de Cabrera (1993) los cuales, de ser eliminados los hace equivalentes al modelo de Stanford \& Smith (1972). En este sentido, los modelos de Broadbent (1986) $\left(\mathrm{R}^{2}=0.925\right)$ seguido por el modelo de Stanford \& Smith (1972) se muestran como los mejores descriptores de la dinámica de mineralización para este suelo $\left(\mathrm{R}^{2}=0.8692\right)$ sin la aplicación de biosólidos como se muestra en la Figura 2.

Estudios presentados por Camargo et al. (2002) y Pereira et al. (2005) indican que el modelo de Broadbent (1986) resultó ser el menos adecuado para describir el proceso de mineralización en suelos representativos de Brasil y Pereira et al. (2005) reportaron como modelo de mejor ajuste el modelo hiperbólico propuesto por Juma et al. (1984), el cual presentó el ajuste más deficiente $\left(\mathrm{R}^{2}=0,30\right)$ según los mostrado en la Tabla 3. Este comportamiento tan diverso pudo ser debido probablemente a las diferencias en los tipos de suelos estudiados y al tiempo y condiciones del ensayo de incubación.

Los resultados de la modelación de la mineralización del nitrógeno del biosólido deshidratado aplicado al suelo se muestran en la Tabla 4.

Para el caso del biosólido deshidratado, los modelos de mejor ajuste fueron en su orden el de Broadbent (1986) $\left(\mathrm{R}^{2}\right.$ $=0,907)$, Jones $(1984)\left(\mathrm{R}^{2}=0,88\right)$ y el modelo polinomial de segundo orden $\left(\mathrm{R}^{2}=0,87\right)$; sin embargo, para el caso del 
A.

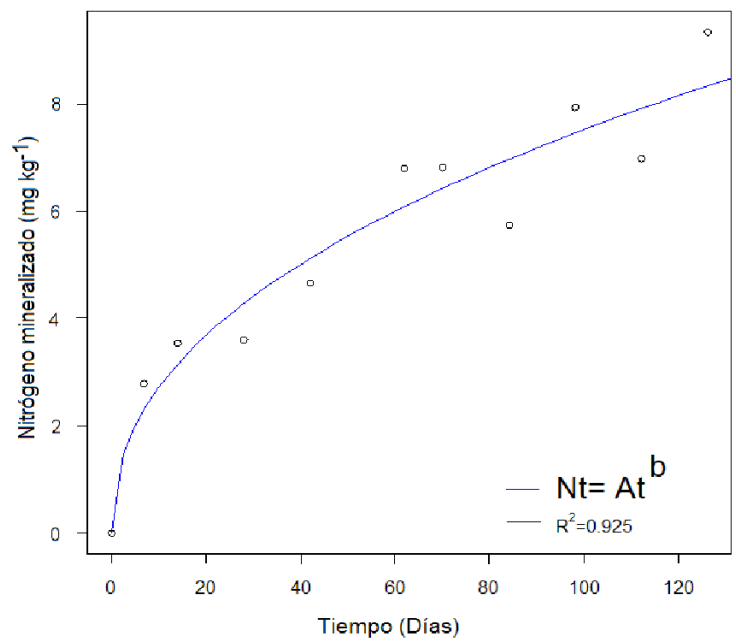

B.

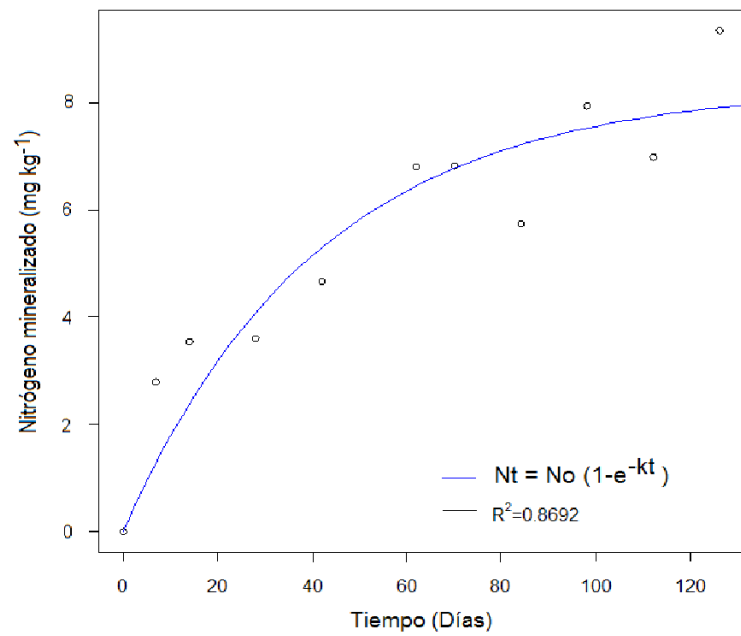

$\mathrm{R}^{2}$-Coeficiente de determinación

Figura 2. Modelos para estimar la mineralización en el tratamiento control. (A) modelo propuesto por Broadbent (1986), (B) modelo de Stanford \& Smith (1972)

Tabla 4. Resultados de la modelación sobre el biosólido deshidratado

\begin{tabular}{|c|c|c|c|c|}
\hline Modelo & Parámetros & Estimación & $\overline{R^{2}}$ & CME \\
\hline a. Stanford \& Smith (1972) & $\begin{array}{c}\mathrm{N}_{0} \\
\mathrm{k}\end{array}$ & $\begin{array}{r}24,1120 \\
0,0240 \\
\end{array}$ & 0,8595 & 9,653 \\
\hline \multirow{3}{*}{ b. Jones (1984) } & $\mathrm{N}_{1}$ & $\star 2,9990$ & \multirow{3}{*}{0,8816} & \multirow{3}{*}{9,150} \\
\hline & $\mathrm{N}_{0}$ & 24,1100 & & \\
\hline & $\mathrm{k}$ & $\star 0,0157$ & & \\
\hline c. Juma et al. (1984) & $\mathrm{N}_{0}$ & 19,8840 & 0,2897 & 25,409 \\
\hline \multirow{2}{*}{ d. Broadbent (1986) } & A & 2,8440 & \multirow{2}{*}{0,9076} & \multirow{2}{*}{6,345} \\
\hline & $\mathrm{b}$ & 0,4430 & & \\
\hline \multirow{2}{*}{ e. Lineal } & $\beta_{0}$ & 5,7440 & \multirow{2}{*}{0,8378} & \multirow{2}{*}{11,140} \\
\hline & $\beta_{1}$ & 0,1650 & & \\
\hline \multirow{3}{*}{ f. Polinomial (2) } & $\beta_{0}$ & *3,8040 & \multirow{3}{*}{0,8733} & \multirow{3}{*}{9,790} \\
\hline & $\beta_{1}$ & 0,2860 & & \\
\hline & $\beta_{2}$ & ${ }^{*}-0,0009$ & & \\
\hline \multirow{2}{*}{ g. Exponencial } & A & 8,2090 & \multirow{2}{*}{0,7624} & \multirow{2}{*}{16,320} \\
\hline & $\mathrm{k}$ & 0,0100 & & \\
\hline
\end{tabular}

A.

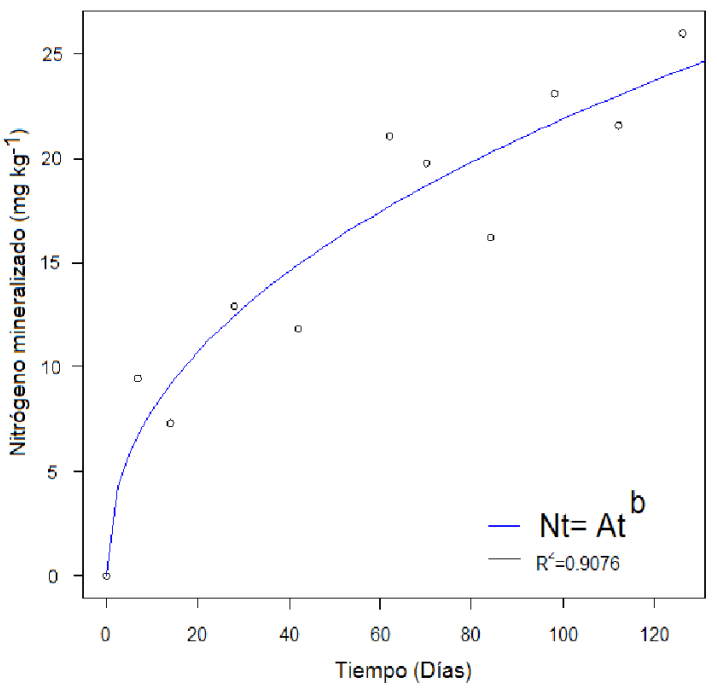

modelo de Jones (1984) la constante aditiva $\mathrm{N}_{1}$ y para el modelo polinomial, la constante $\mathrm{N}_{0}$ y el coeficiente cuadrático $\mathrm{k}_{2}$ son poco significativos, haciéndolos equivalentes a los modelos de Stanford \& Smith (1972) y a un modelo lineal respectivamente. De esta manera, mezclando juicios de bondad de ajuste y significancia de parámetros, el modelo que ofrece un mejor ajuste es el de Broadbent (1986) seguido por el de Standford $\&$ Smith (1972) como se muestra en la Figura 3.

La postulación del modelo de Stanford \& Smith (1972) como herramienta de modelación coincide, aunque con menor bondad de ajuste, con los hallazgos documentados por Boeira \& Maximiliano (2009), quienes los reportan como el modelo de mejor ajuste a sus datos experimentales, encontrando coeficientes de determinación $\left(\mathrm{R}^{2}\right)$ hasta del 0.96. De igual manera, Smith et al. (1998) reportan valores de coeficientes de determinación $\left(\mathrm{R}^{2}\right)$ entre 0.84 y 0.97 .

B.

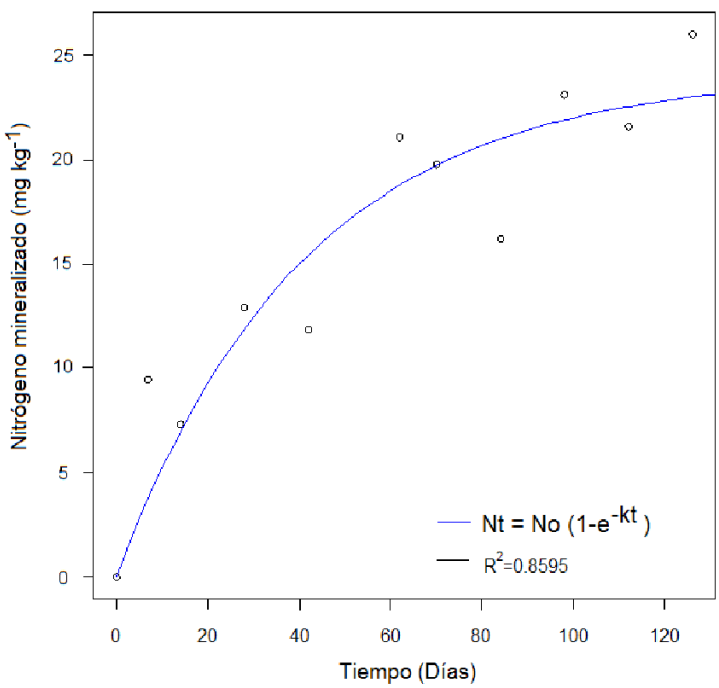

$\mathrm{R}^{2}$-Coeficiente de determinación

Figura 3. Modelos para estimar la mineralización en el suelo aplicando biosólido deshidratado. (A) Modelo propuesto por Broadbent (1986); (B) Modelo de Stanford \& Smith (1972) 
La Tabla 5 muestra los resultados de la modelación de los datos obtenidos del biosólido secado térmicamente.

Tabla 5. Resultados de la modelación del biosólido secado térmicamente

\begin{tabular}{|c|c|c|c|c|}
\hline Modelo & Parámetros & Estimación & $\mathrm{R}^{2}$ & CME \\
\hline a. Stanford \& Smith (1972) & $\begin{array}{c}\mathrm{N}_{0} \\
\mathrm{k}\end{array}$ & $\begin{array}{r}37,75700 \\
0,02000 \\
\end{array}$ & 0,836 & 25,965 \\
\hline b. Jones (1984) & $\begin{array}{l}\mathrm{N}_{1} \\
\mathrm{~N}_{0} \\
\mathrm{k}\end{array}$ & $\begin{array}{r}* 5,32400 \\
* 45,67600 \\
* 0,00920\end{array}$ & 0,865 & 23,893 \\
\hline c. Cabrera (1993) & $\begin{array}{l}\mathrm{N}_{0} \\
\mathrm{k}_{1} \\
\mathrm{k}_{2}\end{array}$ & $\begin{array}{r}10,92700 \\
\star 0,33800 \\
0,21600 \\
\end{array}$ & 0,910 & 16,037 \\
\hline d. Juma et al. (1984) & $\mathrm{N}_{0}$ & 29,02800 & 0,270 & 63,301 \\
\hline e. Broadbent (1986) & $\begin{array}{l}A \\
b\end{array}$ & $\begin{array}{l}3,52000 \\
0,48200 \\
\end{array}$ & 0,894 & 16,693 \\
\hline f. Lineal & $\begin{array}{l}\beta_{0} \\
\beta_{1}\end{array}$ & $\begin{array}{l}7,69700 \\
0,25200 \\
\end{array}$ & 0,852 & 23,340 \\
\hline g. Polinomial (2) & $\begin{array}{l}\beta_{0} \\
\beta_{1} \\
\beta_{2}\end{array}$ & $\begin{array}{r}6,22800 \\
0,34400 \\
*-0,00075 \\
\end{array}$ & 0,861 & 24,680 \\
\hline h. Exponencial & $\begin{array}{l}A \\
k\end{array}$ & $\begin{array}{r}11,20700 \\
0,01000 \\
\end{array}$ & 0,803 & 31,149 \\
\hline
\end{tabular}

* Parámetro no significativo (p-value $>0.05)$

De acuerdo con la Tabla 5, los modelos de mejor ajuste fueron en su orden el de Broadbent (1986) $\left(\mathrm{R}^{2}=0,89\right)$, lineal $\left(\mathrm{R}^{2}=0,85\right)$ y en tercer lugar, con un ajuste aceptable el modelo de Stanford \& Smith $(1972)\left(\mathrm{R}^{2}=0,836\right)$ como lo muestra la Figura 4. Aunque los modelos de Jones (1984), Cabrera (1993) y Polinomial de segundo orden presentaron ajustes importantes $\left(\mathrm{R}^{2}>0,85\right)$, estos modelos presentan parámetros poco significativos, lo cual los hace equivalentes al modelo de Stanford \& Smith (1972) y lineal. El modelo de Juma et al. (1984) presentó el menor ajuste $\left(\mathrm{R}^{2}=0,27\right)$, muy inferior a lo encontrado por Gil et al. (2011) quienes aunque reportaron con este modelo los valores de ajuste más bajos comparados con otros modelos, dichos valores oscilaron entre 0.86 y 0.98 .

A.

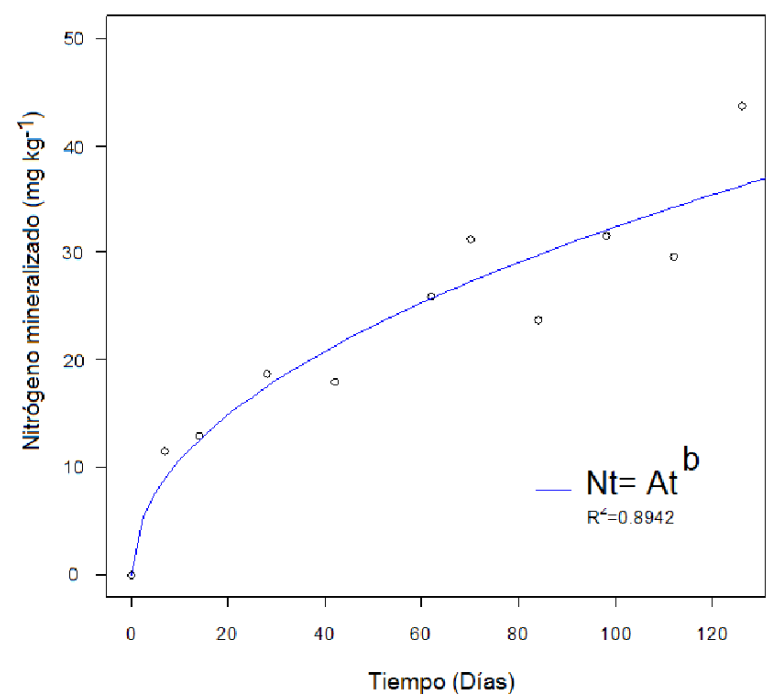

La Tabla 6 muestra los resultados de la modelación de los datos del biosólido alcalinizado.

Tabla 6. Resultados modelación biosólido alcalinizado

\begin{tabular}{|c|c|c|c|c|}
\hline Modelo & Parámetros & Valores & $\overline{R^{2}}$ & CME \\
\hline a. Stanford \& Smith (1972) & $\begin{array}{c}\mathrm{N}_{0} \\
\mathrm{k}\end{array}$ & $\begin{array}{r}18,07200 \\
0,04800\end{array}$ & 0,813 & 8,023 \\
\hline & $N_{1}$ & $\star 2,74100$ & & \\
\hline b. Jones (1984) & $\begin{array}{c}\mathrm{N}_{0} \\
\mathrm{k}\end{array}$ & $\begin{array}{r}{ }^{*} 16,36000 \\
{ }^{*} 0,03100\end{array}$ & 0,832 & 8,093 \\
\hline c. Juma et al. (1984) & $\mathrm{N}_{0}$ & 17,76800 & 0,348 & 11,448 \\
\hline d. Broadbent (1986) & $\begin{array}{l}A \\
b\end{array}$ & $\begin{array}{l}4,37000 \\
0,31300\end{array}$ & 0,898 & 4,353 \\
\hline e. Lineal & $\begin{array}{l}\beta_{0} \\
\beta_{1}\end{array}$ & $\begin{array}{l}6,61500 \\
0,12300\end{array}$ & 0,749 & 10,773 \\
\hline f. Polinomial (2) & $\begin{array}{l}\beta_{0} \\
\beta_{1} \\
\beta_{2}\end{array}$ & $\begin{array}{r}4,86500 \\
0,23200 \\
*-0,00089\end{array}$ & 0,795 & 9,886 \\
\hline g. Exponencial & $\begin{array}{l}\mathrm{A} \\
\mathrm{k}\end{array}$ & $\begin{array}{l}8,18100 \\
0,00800 \\
\end{array}$ & 0,689 & 13,342 \\
\hline
\end{tabular}

* Parámetro no significativo ( $p$-value $>0.05)$

Para este caso nuevamente se observa que los modelos de Boadbent (1986) y Stanford \& Smith (1972) ofrecen la mejor opción de modelación como lo muestra la Figura 5. Estos hallazgos son similares a lo reportado por Flores et al. (2010) quienes encontraron que la mineralización del biosólido alcalinizado aplicado al suelo en dosis de 200 a $600 \mathrm{~kg} \mathrm{ha}^{-1} \mathrm{se}$ ajustaba al modelo propuesto por Stanford \& Smith (1972) para todas las dosis evaluadas. El modelo Juma et al. (1984) mostró los resultados más deficientes en el ajuste, mientras que nuevamente, como en el caso del biosólido deshidratado, el modelo propuesto por Cabrera (1993) no presentó convergencia en la estimación de su parámetros.

La Tabla 7 muestra una comparación de los modelos empleados.

La comparación de la bondad de ajuste de los modelos empleados para las tres calidades de biosólido evaluados y el

B.

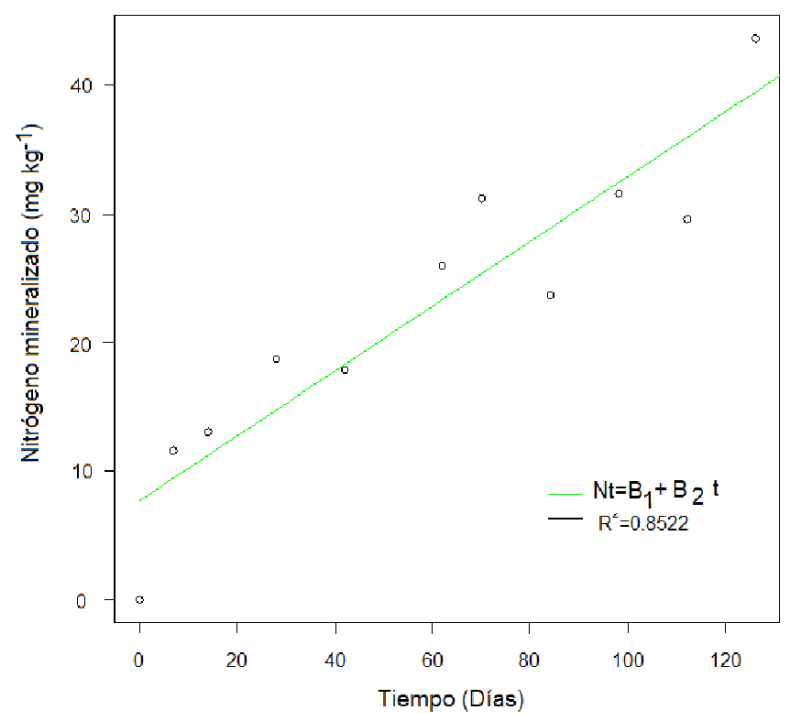

$\mathrm{R}^{2}$-Coeficiente de determinación

Figura 4. Modelos para estimar la mineralización en el suelo aplicando biosólido secado térmicamente. (A) Modelo propuesto por Broadbent (1986); (B) Modelo de Stanford \& Smith (1972) B 
A.

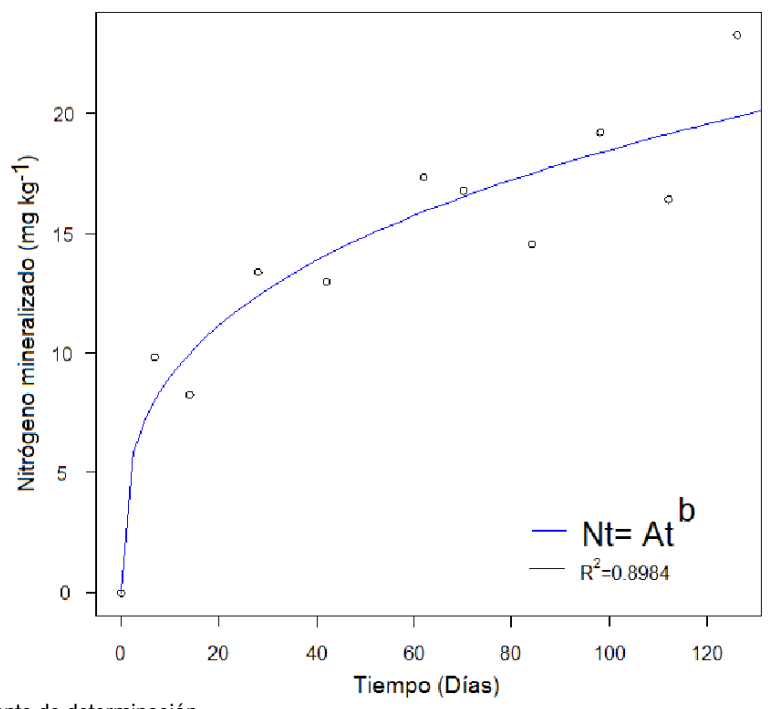

B.

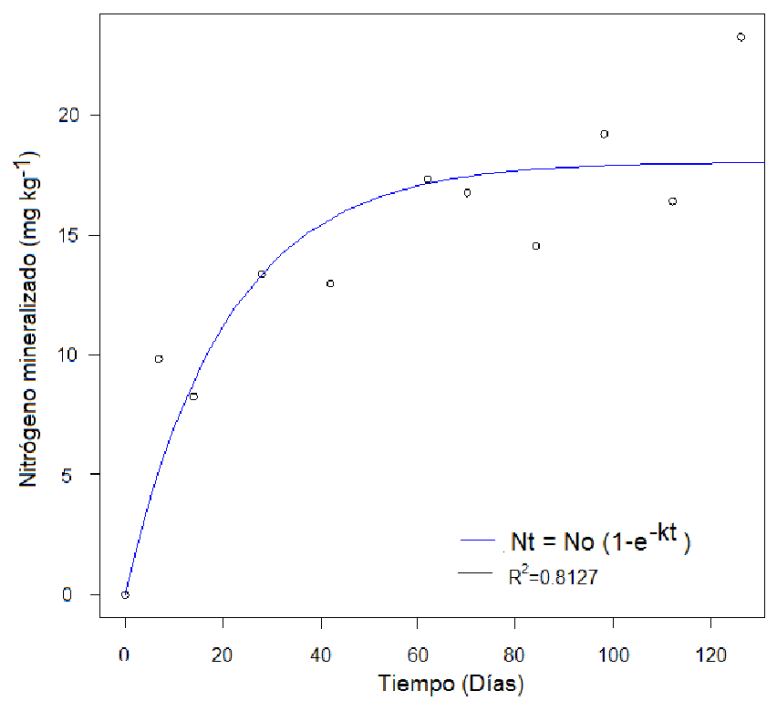

$\mathrm{R}^{2}$-Coeficiente de determinación

Figura 5. Modelos para estimar la mineralización en el suelo aplicando biosólido alcalinizado. (A) Modelo propuesto por Broadbent (1986); (B) Modelo de Stanford \& Smith (1972)

Tabla 7. Coeficiente de determinación $\left(\mathrm{R}^{2}\right)$ para los diferentes modelos y biosólidos evaluados

\begin{tabular}{lcccc}
\hline \multirow{2}{*}{ Modelo } & \multicolumn{4}{c}{ Tratamiento de biosólido } \\
\cline { 2 - 5 } a. Stanford \& Smith (1972) & $0.869^{2}$ & $0.860^{2}$ & $0.836^{3}$ & $0.813^{2}$ \\
b. Jones (1984) & $0.894^{\star}$ & $0.882^{\star}$ & $0.865^{\star}$ & $0.832^{\star}$ \\
c. Cabrera (1993) & $0.932^{*}$ & $\mathrm{~N} / \mathrm{A}$ & $0.910^{\star}$ & $\mathrm{N} / \mathrm{A}$ \\
d. Juma et al. (1984) & 0.301 & 0.290 & 0.270 & 0.348 \\
e. Broadbent (1986) & $0.925^{1}$ & $0.908^{1}$ & $0.894^{1}$ & $0.898^{1}$ \\
f. Lineal & 0.856 & $0.838^{3}$ & $0.852^{2}$ & $0.749^{3}$ \\
g. Polinomial (2) & $0.885^{3}$ & $0.873^{*}$ & $0.861^{*}$ & $0.795^{\star}$ \\
h. Exponencial & 0.786 & 0.762 & 0.803 & 0.689 \\
i. Inubushi et al. (1985) & $\mathrm{N} / \mathrm{A}$ & $\mathrm{N} / \mathrm{A}$ & $\mathrm{N} / \mathrm{A}$ & $\mathrm{N} / \mathrm{A}$ \\
\hline
\end{tabular}

N/A: no presentó ajuste; ${ }^{*}$ Con parámetros no significativos (p-value $\left.>0.05\right) ;{ }^{1}$ Modelo con mejor ajuste; ${ }^{2}$ Segundo Mejor ajuste; ${ }^{3}$ Tercer mejor ajuste; Con. - Control; Des. - Deshidratado; Alca. - Alcalino

tratamiento control muestra que en todos los casos el modelo parabólico propuesto por Broadbent (1986) representó el mejor ajuste. Adicionalmente, se puede resaltar al modelo de Stanford y Smith (1972) como una alternativa de modelación, ya que en tres de los cuatro casos evaluados representó la segunda opción de modelación, lo que hace posible utilizar dicho modelo determinando una medida de la velocidad de mineralización $\left(1-\mathrm{e}^{-\mathrm{kt}}\right)$.

De otro lado, el modelo de Cabrera (1993) no presentó convergencia en la estimación de sus parámetros para los datos correspondientes a los biosólidos deshidratado y alcalinizado, como tampoco la presentó el modelo de Inubushi et al. (1985) en ninguno de los tratamientos evaluados, lo cual puede deberse a lo planteado por Dou et al. (1996) quienes recomiendan que los modelos doble exponencial pueden emplearse sobre ensayos con duración de 30 semanas de seguimiento.

Autores como Camargo et al. (2002) y Pereira et al. (2005) reportaron que para los suelos de Brasil, los modelos de Standford \& Smith, Jones (1984), Cabrera (1993), Inubushi et al. (1985), Juma et al. (1984) y Broadbent (1986) pueden describir la mineralización de dichos suelos con coeficientes de determinación $\left(\mathrm{R}^{2}\right)$ por encima de 0.95 . Sin embargo, estos resultados consideran la aplicación de fuentes de materia orgánica como la empleada en esta investigación, lo cual afecta el proceso de mineralización en el suelo.

Con el objetivo de evaluar el efecto de los diferentes tratamientos aplicados al biosólido sobre la dinámica de mineralización en el suelo, la Tabla 8 presenta una comparación de los parámetros estimados para el modelo parabólico de Broadbent (1986) y simple exponencial de Stanford y Smith (1972).

Tabla 8. Parámetros estimados para los modelos parabólico y simple exponencial según tipo de biosólido

\begin{tabular}{lccccc}
\hline \multirow{2}{*}{ Tratamiento } & \multicolumn{2}{c}{ Broadbent (1986) } & & \multicolumn{2}{c}{ Stanford \& Smith (1972) } \\
\cline { 2 - 3 } \cline { 5 - 6 } Control & $\mathbf{A}$ & $\mathbf{b}$ & & $\mathbf{N}_{\mathbf{0}}$ & $\mathbf{k}$ \\
Deshidratado & 0,977 & 0,443 & & 8,29 & 0,024 \\
Secado Térmicamente & 2,844 & 0,443 & & 24,11 & 0,024 \\
Alcalinizado & 3,520 & 0,482 & & 37,76 & 0,020 \\
\hline
\end{tabular}

Vale la pena resaltar las posibilidades de interpretación que brinda el modelo de Stanford \& Smith (1972) desde el cual es posible deducir, a través de la comparación de las estimaciones del parámetro $\mathrm{N}_{0}$, que el suelo experimental sobre el cual se realizó el ensayo, presenta un potencial máximo de mineralización de $8,29 \mathrm{mg} \mathrm{kg}^{-1}$, el cual se incrementa cuando se aplica el biosólido deshidratado $\left(\mathrm{N}_{0}=24,11\right)$, potencial que logra mejorarse aún más con la aplicación del biosólido secado térmicamente $\left(\mathrm{N}_{0}=37,76\right)$. En el biosólido alcalinizado, aunque hubo un aumento comparado con el tratamiento control, éste fue inferior $\left(\mathrm{N}_{0}=18,07\right)$ al identificado para el biosólido deshidratado y el secado térmicamente, corroborando lo encontrado por Rigby et al. (2009) quienes encontraron que el proceso de secado térmico influye en la mineralización del nitrógeno. En general se demuestra que la aplicación de biosólidos incrementa el contenido de nitrógeno orgánico en el suelo, lo que aumenta la mineralización de dicho nitrógeno. 


\section{Conclusiones}

1. Los resultados de la investigación mostraron que el modelo propuesto por Broadbent (1986) fue el que mejor se ajustó para describir el proceso de mineralización del suelo Vertic Endoaquepts evaluado y predecir la transformación del nitrógeno orgánico contenido en los biosólidos aplicados en nitrógeno mineralizado disponible para las plantas seguido por el modelo de Stanford \& Smith (1972).

2. El modelo de Stanford \& Smith (1972) permitió corroborar el incremento del nitrógeno orgánico $\mathrm{N}_{0}$ cuando se realiza la aplicación de biosólidos, siendo mayor la mineralización con relación al tratamiento testigo independiente de las características del biosólido.

3. Los modelos reportados por Jones (1984), Cabrera (1993) e Inubushi et al. (1985) no presentaron convergencia en la estimación de sus parámetros para todos los tratamientos.

\section{Agradecimientos}

Los autores agradecen a Colciencias y a la Universidad del Valle por su apoyo financiero en el proyecto código 1106-48925147 y por la financiación del estudiante Jorge Silva como becario de doctorado nacional.

\section{Literatura Citada}

Bettiol, W.; Camargo, O. A disposição de lodo de esgoto em solo agrícola. In: Bettiol, W.; Camargo, O. Lodo de esgoto impactos ambientais na agricultura. Jaguariúna: EMBRAPA, 2.ed. Cap.2, 2008. p.25-36.

Boeira, R. C. Uso de lodo de esgoto como fertilizante orgânico: Disponibilização de nitrogênio em solo tropical. Jaguariúna: EMBRAPA, 2004. 3p. Comunicado Técnico, 12

Boeira, R. C. Lixiviação de nitrogênio em Latossolo incubado com lodo de esgoto. Revista Brasilera de Ciencias do Solo, v33, p.947-958, 2009.

Boeira, R. C.; Maximiliano, V. C. Dinâmica da mineralizaçao de nitrogênio de lodos de esgoto. In: Bettiol, W.; Camargo, O. Lodo de esgoto impactos ambientais na agricultura. Jaguariúna: EMBRAPA, 2.ed. Cap.7, 2008. p.125-136.

Boeira, R. C.; Maximiliano, V. C. Mineralização de compostos nitrogenados de lodos de esgoto na quinta aplicação em Latossolo. Revista Brasilera de Ciencias do Solo, v33, p.711-722, 2009.

Broadbent, F. E. Empirical modeling of soil nitrogen mineralization. Soil Science, v.141, p.208-213, 1986.

Cabrera, M. L. Modeling the flush of nitrogen mineralization caused by drying and rewetting soils. Soil Science Society of America Journal, v.57, p.63-66, 1993.

Cabrera, M. L.; Kissel, D. E.; Vigil, M. F. Nitrogen mineralization from organic Residues: Research opportunities. Jounal Environmental Quality, v34, p75-79, 2005.

Camargo, F. A.; Gianello, C.; Tedesco, M. J.; Riboldi, J.; Meurer, E. J.; Bissani, C. A. Empirical models to predict soil nitrogen mineralization. Ciência Rural, v.32, p.393399, 2002.
CETESB - Companhia Ambiental do Estado de São Paulo. Aplicação de lodos de sistemas de tratamento biológico em áreas agrícolas - Critérios para projeto e operação. São Paulo, 1999. 32p. Manual Técnico, 230

Dynia, J. F.; de Souza, M. D.; Boeira, R. C. Lixiviação de nitrato em Latossolo cultivado com milho após aplicações sucessivas de lodo de esgoto. Pesquisa Agropecuária Brasileira, v41, p.855-862, 2006.

Dou, Z.; Toth, J. D.; Jabro, J. D.; Fox, R. H.; Fritton D. D. Soil nitrogen mineralization during laboratory incubation: dynamics and model fitting. Soil Biology and Biochemestry, v.28, p.625-632, 1996.

Flores, M. J. P.; Acosta, M. Z. P.; Salas, G. E.; Pérez, F. C.; Corral, A. A. Y.; Salazar, S. E.; Trejo, E. H. I. Mineralización de nitrógeno en biosólidos estabilizados con cal. Revista Terra Latinoamericana, v.28, p.307-317, 2010.

Flores-Márgez, J. P.; Corral-Díaz, B.; Sapien-Mediano, G. Mineralización de nitrógeno de biosólidos estabilizados con cal en suelos agrícolas. Revista Terra Latinoamericana, v.25, p.409-417, 2007.

Franco, H. O.; Mckelligan, G. A.; López, O. A.; Espinosa, C. F.; Escamilla, S. E. Dynamics of carbon, nitrogen and phosphorus in soil amended with irradiated, pasteurized and limed biosolids. Bioresource Technology, v.87, p.93$102,2003$.

Gil, M. V.; Carballo, M. T.; Calvo, L. F. Modelling N mineralization from bovine manure and sewage sludge composts. Bioresource Technology, v.102, p.863-871, 2011.

Hernandez, T.; Moral, R.; Perez-Espinoza, J.; Moreno-Caselles, M. D.; Gracia, C. Nitrogen mineralization potencial in calcareous soil amended with sewage sludge. Bioresource Technology, v.83, p.213-219, 2002.

Inubushi, K.; Wada, H.; Takai, Y. Easily decomposable organic matter in paddy soil. VI kinetics of nitrogen mineralization in submerged soils. Journal of Soil Science and Plant Nutrition, v.31, p.563-572, 1985.

Jones, A. Estimation of an active fraction soil nitrogen. Communications in Soil Science and Plant Analysis, v.15, p.23-32, 1984.

Juma, N.G.; Paul, E.A.; Mary, B. Kinetic analysis of net mineralization in soil. Soil Science Society of America Journal, v.48, p.753-757, 1984.

Kieft, T.L.; Soroker, E.; Firestone, M. K. Microbial biomass response to a rapid increase in water potential when dry soil is wetted. Soil Biology and Biochemistry. v.19, p.119126, 1987.

Molina, J. A. E.; Clapp, C. E.; Larson, W.E. Potentially mineralizable nitrogen in soil: the simple exponential model does not apply for the first 12 weeks of incubation. Soil Science Society of America Journal, v.44, p.442- 443, 1980.

Ortega, B, R.; Mardonez, R. Variabilidad espacial de la mineralización de nitrógeno de un suelo volcánico de la provincia de Ñuble, VIII región Chile. Revista Agricultura Técnica, v.65, p.221-231, 2005.

Pereira, J. M.; Muniz. J. A.; Silva, C A. Nonlinear models to predict nitrogen mineralization in an oxisol. Scientia Agricola, v.62, p.395-400, 2005. 
Rigby, H.; Perez-Viana, F.; Cass, J.; Rogers, M.; Smith, S. $\mathrm{R}$. The influence of soil and biosolids type, and microbial immobilization on nitrogen availability in biosolids -Amended agricultural soils - Implications for fertilizer recommendations. Soil Use and Management, v25, p.395-408, 2009.

Silva, J. A.; Torres, P.; Cardoza, Y. Thermal drying and alkaline treatment of biosolids: Effects on nitrogen mineralization. Clean-Soil, Air, Water, v.41, p.1-6, 2012.

Smith, S. R.; Durham, E. Nitrogen release and fertiliser value of thermally-dried biosolids . Journal CIWEM, v.16, p.121126, 2002.
Smith S. R.; Woods, V.; Evans T. D. Nitrate dynamics in biosolidstreated soils. III. Significance of the organic nitrogen, a twin-pool exponential model for nitrogen management and comparison with the nitrate production from animal wastes. Bioresource Technology, v.66, p.161-174. 1998.

Stanford, G.; Smith, S. J. Nitrogen mineralization potentials of soil. Soil Science Society of America Journal, v.36, p.465-471, 1972.

Wang, W. J.; Smith, C. J.; Chen, D. Predicting soil nitrogen mineralization dynamics with a modified double exponential model. Soil Science Society of America Journal. v.68 p.1256-1265, 2004. 